\title{
Régions, régionalisation et développement au Québec : quel bilan 50 ans après les premiers découpages administratifs
}

\author{
Martin Simard ${ }^{1}$ \\ Université du Québec à Chicoutimi
}

\section{INTRODUCTION}

La région se présente à nouveau comme un objet d'intérêt pour les chercheurs, quelle que soit l'échelle géographique considérée.

La région est un concept important en géographie, notamment dans la tradition géographique francophone ${ }^{2}$. Selon cette tradition, la région a été décrite comme une unité territoriale presque naturelle produite par la géographie et l'histoire. Cette conception «objectivée» et réductrice a contribué à dévaloriser ce champ d'études, dans la deuxième moitié du $\mathrm{XX}^{\mathrm{e}}$ siècle ${ }^{3}$. Depuis lors, on semble assister à un « retour de la région ", notamment sous le regard d'approches culturaliste, critique ou croisées ${ }^{4}$. Ce mouvement touche divers champs des sciences humaines et sociales. Malgré les flux et les réseaux qui caractérisent la société moderne avancée, la région se présente à nouveau comme un objet d'intérêt pour les chercheurs, quelle que soit l'échelle géographique considérée. Par ailleurs, celle-ci n'a jamais perdu de sa pertinence pour les administrateurs de l'État. À cet égard, mentionnons les débats sur le redécoupage des régions en France ${ }^{5}$. Certains vont jusqu'à dire que la région, ainsi que toutes les formes de localisme et de régionalisme, constituent un rempart contre les effets homogénéisateurs de la mondialisation des marchés et de la culture ${ }^{6}$.

En effet, la région se présente comme une échelle d'analyse signifiante socialement et économiquement car on y étudie les «bassins de vie » des populations, soit l'espace que côtoient les individus au cours de leurs activités quotidiennes ou occasionnelles. Sur le plan politique, la région facilite la gestion des services publics et elle peut servir de socle aux stratégies d'équilibre spatial ou de décentralisation politique ${ }^{7}$. Parler de région ou de régionalisation, c'est s'attarder à la question des délimitations ou des découpages géographiques ou territoriaux infranationaux. En effet, en dépit de certains traits homogènes des territoires régionaux, leurs frontières sont généralement difficiles à tracer car les caractéristiques du milieu adoptent rarement des discontinuités clairement définies ${ }^{8}$. De plus, la question de l'échelle de référence se pose avec acuité car la région ne constitue qu'un palier d'un système spatial multiscalaire qui semble organiser le monde ${ }^{9}$. Finalement, l'enjeu de la marge de manœuvre accordée aux instances politiques fréquemment associées aux régions est un sujet sensible qui doit inévitablement être traité par les États-nations.

Dans cette optique, ce texte vise à faire le point sur la régionalisation et le développement territorial au Québec, près de 50 ans après les premiers découpages administratifs. À l'intérieur de ce demi-État membre de la fédération canadienne, des régions administratives ont été créées par le gouvernement provincial à la fin des années soixante, dans le contexte de mise en place de l'État-providence ${ }^{10}$. Étant maintenant au nombre de 17 , celles-ci servent notamment à localiser les employés de l'État et à gérer certains services sur une base territoriale (transports, ressources naturelles, etc.). En parallèle, des instances micro-régionales ont été constituées à la fin des années 1970, soit les municipalités régionales de comté (MRC). Plusieurs questions découlent de ces gestes d'appropriation territoriale: Quelle doit être l'échelle géographique pertinente? Quels sont les critères de délimitation utilisés? Comment expliquer les divergences de tailles et de populations entre les différentes régions? Pourquoi avoir institué des micro-régions en ajout aux macro-régions déjà 
existantes? Quels pouvoirs sont ou devraient être dévolus aux diverses instances territorialisées? Quel est le sens de la réforme actuelle qui favorise les MRC au détriment des régions adminis-

\section{TERRITOIRES, RÉGIONS ET FRONTIÈRES}

Les termes espace et territoire sont souvent utilisés comme synonymes alors qu'ils présentent des différences aux yeux des géographes. Pour plusieurs, l'espace est une étendue neutre sur laquelle se déploie les activités humaines, alors que le territoire réfère plutôt à un endroit précis ayant une personnalité et des délimitations (le Québec, la NouvelleAngleterre, 1'Andalousie, etc. ${ }^{11}$. Pour d'autres, «l'espace est physique; le territoire est l'espace humanisé $»^{12}$. Selon cette deuxième interprétation, l'espace «...est la réalité matérielle préexistante à toute connaissance et à toute pratique $»^{13}$ alors que le territoire constitue un système de relations des sociétés à l'espace ${ }^{14}$. L'expression territoire apparaît beaucoup plus appropriée en géographie régionale car il s'agit généralement de faire le portrait synthétique d'unités géographiques circonscrites et marquées par la présence humaine.

Le concept de territoire s'apparente fortement à celui de région. Cependant, le territoire n'induit pas d'échelle géographique particulière et la question de la gouvernance n'y est pas directement associée, la plupart du temps ${ }^{15}$. De son côté, la région est habituellement réservée pour traiter de l'échelle infranationale et la dimension politique lui est intrinsèquement reliée. En effet, l'origine latine du terme région n'est-elle pas regere qui signifie régir ou gérer ${ }^{16}$. À travers l'histoire, les empires, royaumes et états ont presque toujours été découpés administrativement ou culturellement en unités intermédiaires caractérisées par le milieu naturel et le genre de vie des populations, à l'image des satrapies de l'empire perse ${ }^{17}$. Les États-nations modernes sont également divisés en régions ${ }^{18}$, que ce soit pour reconnaître des variations mineures de la culture nationale ou pour accommoder une diversité ethnoculturelle inscrite dans la physionomie ou la constitution de l'État. Ces unités constituent des régions même si d'autres termes sont aussi utilisés pour les tratives? Nous tenterons de répondre à ces interrogations par le biais d'une revue de littérature et d'une analyse des politiques actuelles en matière de développement régional.

désigner (département en France, canton en Suisse, comté aux États-Unis, gouvernorat en Tunisie, oblast en Russie, etc.).

Les régions sont donc des territoires de tailles moyennes à l'intérieur d'un pays, lesquels se distinguent des milieux avoisinants sur la base de certains critères alors que la régionalisation réfère à l'art de découper des régions dans un cadre infranational ${ }^{19}$. D'ailleurs, «toute modification des contours de ces territoires, tout projet de regroupement, même pour des raisons purement techniques, deviennent des enjeux politiques $»^{20}$. On peut identifier cinq types de régions ${ }^{21}$, chacune ayant son ou ses critères de définition qui guident la main du cartographe: la région homogène (aussi appelée historique ou culturelle), la région fonctionnelle (également nommée économique ou polarisée), la région politico-administrative, la région d'ancrage et d'action de même que la région "problèmeprogramme-plan » (PPP). À notre avis, la région politico-administrative et la région PPP peuvent être réunies pour ne former qu'une seule catégorie. Une véritable région au sens sociologique ou géographique du terme serait un territoire qui cumule plusieurs de ces variables.

Le critère du substrat social apparaît comme un aspect des plus importants. En effet, les régions sont des territoires où l'on retrouve fréquemment une dynamique socioculturelle particulière qui s'est développée à travers l'histoire, entre individus en situation de co-présence, au sein d'un cadre naturel spécifique. On doit y observer une dynamique de milieu et des pratiques de gouvernance, par opposition à la simple convergence en un lieu d'échanges en réseaux, pour que la région soit davantage qu'un «no man's land $»^{22}$. Le paysage peut être situé comme étant un élément primordial du concept de région, soit la partie visible du phénomène qui permet son «objectivation». Néanmoins, il ne faut pas oublier l'influence des phénomènes naturels et humains qui sont moins 
apparents mais qui demeurent actifs selon des rythmes variés (cycles économiques, crises politiques, changements climatiques, etc.). Ces phénomènes, en particulier ceux d'ordre économique, peuvent s'opérer à des échelles beaucoup plus vastes que celle de la région.

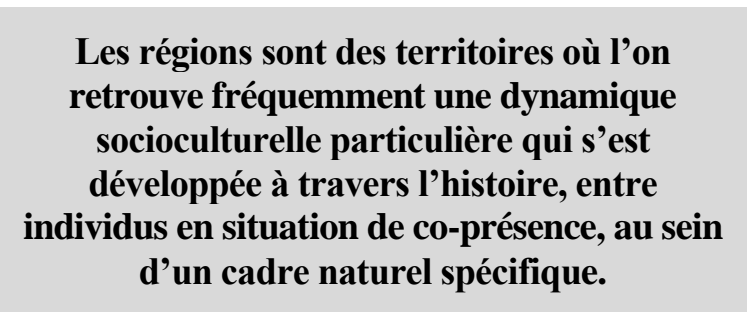

Malgré certains traits homogènes du territoire régional sur les plans social et paysager, les frontières régionales sont généralement difficiles à tracer car les caractéristiques naturelles des milieux et, dans une moindre mesure, les caractéristiques humaines, se transforment progressivement à mesure que l'on se déplace dans l'espace ${ }^{23}$. La région se présente donc davantage comme étant un noyau central entouré d'aires de transition que comme un espace

\section{LA RÉGION À TRAVERS LE TEMPS}

\section{Le développement des villes petites et moyennes ou d'immenses métropoles aboutit au façonnement d'espaces identitaires distincts des régions culturelles qui les accueillent, nommément le quartier ou l'agglomération.}

À l'intérieur du système-monde, la région peut occuper une place plus ou moins prégnante, selon les époques. Il y a quelques siècles, les sociétés rurales étaient largement fondées sur l'agriculture. Les régions se caractérisaient alors par les types d'agriculture ou d'élevage que l'on y exerçait $^{27}$. Celles-ci dépendaient à leur tour du relief, de la nature des sols et du degré d'ensoleillement du milieu. Le tracé des parcelles agricoles était également influencé par les formes $\mathrm{du}$ terrain $^{28}$. Les activités économiques, le paysage et le rythme de vie étaient ainsi en bonne partie dictés par la nature et les saisons. De plus, les cultures et traditions régionales prenaient largement racine dans le territoire, par exemple dans isotrope doté partout des mêmes qualités, à l'instar des formations sociospatiales de Guy Di Méo ${ }^{24}$. Ainsi, toute frontière est simpliste et réductrice de la réalité géographique. À cet égard, les rivages lacustres ou continentaux, les rivières ou la ligne de crêtes de chaînes de montagnes se révèlent des limites conventionnelles faciles à cartographier et à mémoriser. Malgré tout, les limites administratives sont utiles, voire indispensables, pour l'application de lois ou de règles administratives ${ }^{25}$. Une fois établies, ces lignes de démarcation du politique ont tendance à créer de l'homogénéité sur l'espace ainsi borné.

D'une manière plus fondamentale, Raffestin ${ }^{26}$ souligne le fait que toute action humaine non délimitée par une frontière tend à se dissoudre dans l'espace. Bref, la frontière est intimement liée à l'appropriation et au marquage de l'espace, de même qu'à la construction de territoires, de communautés, de sociétés. Plus pratiquement, la région est pour l'État le maillon préférentiel de l'élaboration et de la mise en œuvre des plans d'aménagement et de développement.

l'architecture vernaculaire et ses matériaux de même que dans les habitudes alimentaires des populations, voire quelques modes d'expression de la culture.

Avec les changements socioéconomiques découlant de l'industrialisation et de l'urbanisation aux $\mathrm{XIX}^{\mathrm{e}}$ et $\mathrm{XX}^{\mathrm{e}}$ siècles, les régions ont été soumises à des processus significatifs de transformations. Dès lors, les industries locales peuvent façonner des produits qui ne sont aucunement liés aux ressources du milieu ${ }^{29}$. Les réseaux de transport et de communication favorisent également les échanges entre des lieux situés à grande distance. De surcroit, les médias et le tourisme amènent les gens à mieux connaître ce qui se passe ailleurs. L'émigration, l'immigration et la mobilité généralisée conduisent à la diversification des populations sur tous les territoires. Le développement des villes petites et moyennes ou d'immenses métropoles aboutit au façonnement d'espaces identitaires distincts des régions culturelles qui les accueillent, nommément le quartier ou l'agglomération. 
Les villes de grande taille deviennent des «portails » accédant à des réseaux physiques ou virtuels diffusant une culture nationale ou des bribes de cultures «étrangères $»^{30}$. Cela permet l'émergence de valeurs et de pratiques contrastant avec les traditions régionales. Ce n'est pas un hasard si la géographie régionale classique éprouvait un malaise face à la ville, cet objet étrange et dynamique qui semblait briser l'harmonie des paysages et de la vie en société propres aux espaces régionaux des sociétés agraires traditionnelles ${ }^{31}$. Les sciences sociales furent ainsi dominées par l'écologie urbaine des sociologues américains sur une grande partie du $\mathrm{XX}^{\mathrm{e}}$ siècle $^{32}$ avant que les études régionales ne redeviennent populaires comme angle d'analyse des relations espaces-sociétés ${ }^{33}$.

Tous ces changements technologiques et sociologiques produisent une certaine standardisation de la culture ainsi que la disparition progressive de plusieurs régionalismes. Dans ce contexte, la question de l'existence ou non des régions se pose au sein des sociétés modernes avancées ${ }^{34}$. À notre époque, la vie des individus se ressemble beaucoup, du moins à l'intérieur de grandes aires civilisationnelles. En Occident, quelle que soit sa région d'appartenance ou de résidence, on retrouve généralement un habitat sous forme de bungalow, une culture musicale standardisée, un engouement

\section{BREF PORTRAIT DES RÉGIONS DU QUÉBEC}

Au Québec, la présence millénaire des autochtones a été suivie de quatre siècles de peuplement d'origine principalement française puis britannique et irlandaise ${ }^{39}$. La colonie française de la vallée du Saint-Laurent est l'un des premiers noyaux de peuplement européen du continent nord-américain ainsi que le berceau du Québec et du Canada ${ }^{40}$. Le Québec connaîtra une expansion progressive de son écoumène, de l'axe du fleuve Saint-Laurent vers l'arrière-pays, notamment avec les plans de colonisation agricole de la fin du XIX ${ }^{\mathrm{e}}$ siècle et du début $\mathrm{du} \mathrm{XX}^{\mathrm{e} 41}$. À la suite des ajustements de frontières de 1912 (gain de l'Ungava) et 1927 (perte du Labrador), le Québec occupe la presque totalité de la péninsule du Québec-Labrador, un territoire de 1,5 million de $\mathrm{km}^{2}$. Néanmoins, l'écoumène, défini ici comme l'espace municipalisé, se concentre dans pour les jeux électroniques, les mêmes chaînes de restauration rapide, etc. Les traditions régionales cèdent de plus en plus la place à des pratiques universelles ou à des habitudes calquées sur la classe sociale, sur l'âge ou sur le style de vie ${ }^{35}$. Les forces homogénéisatrices de la mondialisation tentent aujourd'hui de gommer les héritages culturels régionaux ou nationaux et ils y réussissent dans une large mesure.

En dépit de l'industrialisation, de l'urbanisation et de la mondialisation, il semble que la région conserve une résonnance considérable chez les populations. Les territoires du quotidien, les gens que l'on connaît ou les équipements et services sont organisés sur une base locale ou régionale. Des phénomènes comme les fermetures d'usines, l'abolition de services publics ou la migration des jeunes amènent souvent une réaction des populations, lesquelles expriment ainsi leur attachement à la région et à sa pérennité ${ }^{36}$. L'appartenance régionale n'est plus le principal facteur explicatif des comportements sociaux et des valeurs des individus, mais le sentiment d'appartenance régional participe à l'identité individuelle et à l'intégration sociale des personnes $^{37}$. Le milieu régional est aussi un lieu important d'exercice de la citoyenneté par le biais des débats et discussions sur le cadre de vie ou la qualité de l'environnement ${ }^{38}$.

la partie sud de la province et il ne compte que pour $13,7 \%$ de la superficie du Québec ${ }^{42}$. De surcroît, plus de $90 \%$ du territoire est de propriété publique. En conséquence, la densité de population n'est que de $6,3 \mathrm{~h} / \mathrm{km}^{2}$, quoique celle-ci grimpe à $45,7 \mathrm{~h} / \mathrm{km}^{2}$ lorsque l'on utilise uniquement l'écoumène pour son calcul.

En dépit d'un peuplement européen assez récent, le Québec se régionalisera progressivement, des portions de territoires prenant le nom des villes qui les dominent (Montréal ou Capitale-Nationale), de leur position par rapport au fleuve (Bas-SaintLaurent ou Côte-Nord) ou des affluents qui se déversent dans le Saint-Laurent (Outaouais, Mauricie, Saguenay-Lac-Saint-Jean, etc. $)^{43}$. En plus d'être des espaces culturels et économiques, les 
régions du Québec sont des territoires politiques ou administratifs délimités précisément par le gouvernement provincial. Au Québec, les régions administratives ont été mises en fonction par ce palier de gouvernement, en $1966^{44}$. Celles-ci s'inspiraient de la proposition de Benoît Brouillette, faite en 1959, et d'études sur les aires de polarisation de Montréal, Québec et des villes moyennes de la province ${ }^{45}$. Au nombre de dix à ce moment, elles ont été redécoupées en 1987 et 1993 pour atteindre le nombre de 17 (voir la carte 1). Il s'agit de grands territoires qui regroupent, dans plusieurs cas, non pas une seule mais plusieurs régions historiques ou culturelles, par exemple l'Abitibi et le Témiscamingue ou le Saguenay et le Lac-Saint-Jean.

Les régions administratives québécoises servent à la planification gouvernementale (projets routiers, exploitation de la forêt sur les terres publiques, etc.) et à fournir des services publics aux citoyens sur une base territoriale (émission de permis, attribution de baux de villégiature, etc. $)^{46}$. Leur fonction demeure assez modeste si on la compare aux recommandations du rapport de la Commission La Haye, déposé en 1968, et au contenu de l'avant-projet de loi sur l'urbanisme et l'aménagement du territoire soumis à l'Assemblée nationale en $1972^{47}$. La population totale résidant sur le territoire semble être le principal critère de délimitation des frontières, la population moyenne des régions étant de 483216 en 2014, pour une superficie moyenne de $77184 \mathrm{~km}^{2}$. De plus, dix régions administratives sur 17 affichent une population se situant entre 200000 et $600000 \mathrm{~h}$ (voir le tableau 1). Considérant le fait que la densité de population n'est pas la même partout, la taille des régions administratives varie énormément de manière à regrouper des masses critiques de citoyens. En fait, il y a une relation inversement proportionnelle entre la superficie des régions et leur population totale. Ainsi, la plus petite région couvre à peine $246 \mathrm{~km}^{2}$ (territoire de l'Île-Jésus communément appelé Laval) alors que la plus grande fait $718229 \mathrm{~km}^{2}$ (Nord-duQuébec), soit près de $50 \%$ du territoire québécois.

\section{Carte 1 - Carte des régions administratives du Québec ${ }^{48}$}

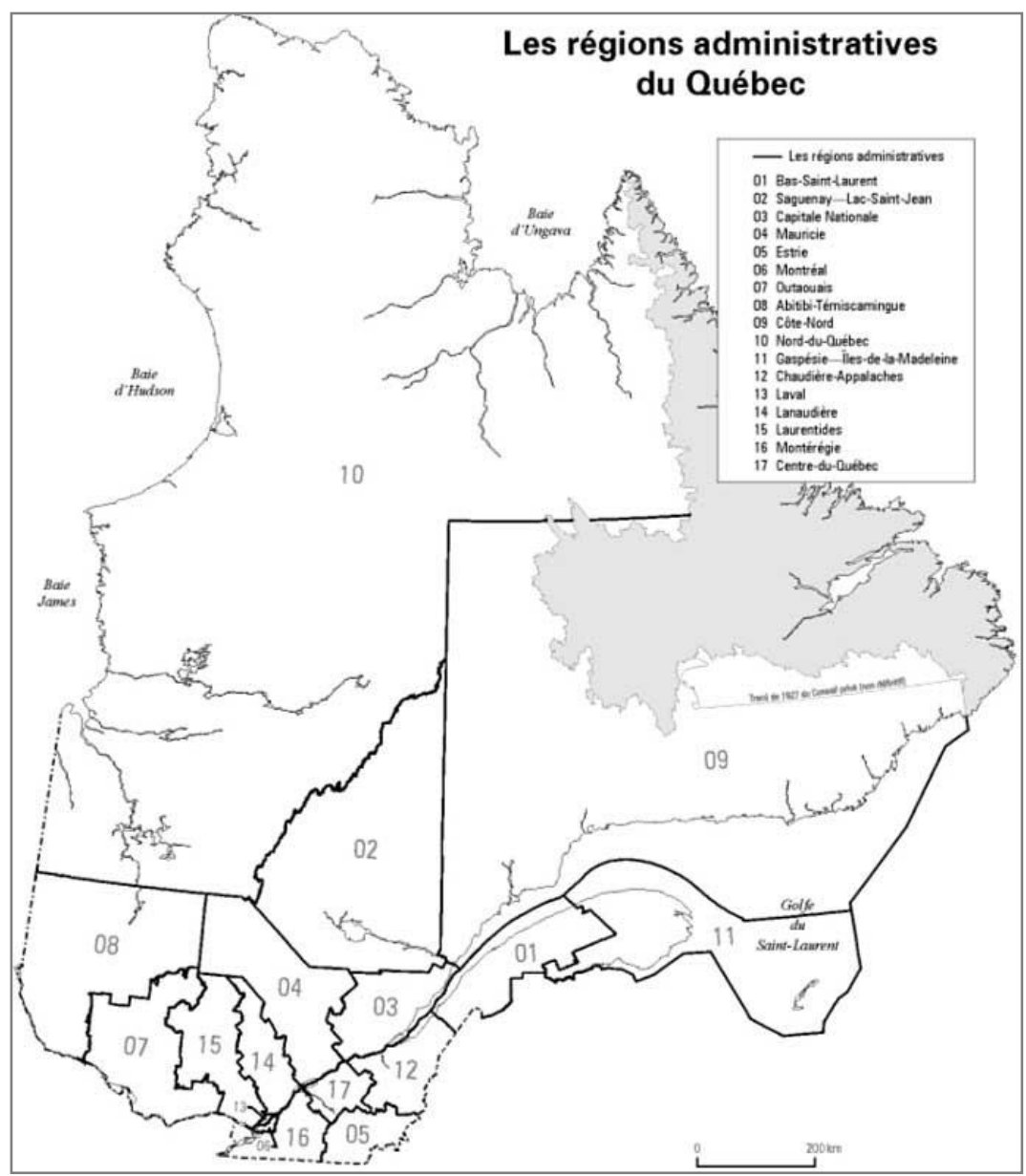

(Source : http://www.tlfq.ulaval.ca/axl/amnord/quebec-Regions_admin-carte.htm) 
Les régions administratives québécoises ne sont pas établies sur la base d'attributs naturels, sauf la Gaspésie, qui forme une péninsule, de même que Laval et Montréal qui reposent sur des îles. Même si leur territoire est occasionnellement centré sur une rivière ou un lac (Mauricie, Outaouais, Saguenay-Lac-Saint-Jean), les frontières régionales coupent sans grandes considérations les trois régions physiographiques, soit le Bouclier canadien, les basses terres du Saint-Laurent et de la Baie d'Hudson ainsi que le Massif appalachien (voir la carte 2). La carte du Québec administratif ne tient pas davantage compte des zones climatiques et de végétation. En outre, les parallèles et méridiens sont quelquefois utilisés comme points de repère ${ }^{49}$, pratique qui n'est jamais signe d'un respect des particularités du terrain, tant pour les espaces régionaux que pour les territoires provinciaux ou nationaux ${ }^{50}$. Néanmoins, la ligne de partage des eaux entre les bassins versants de la Baie d'Hudson et du Saint-Laurent a été mise à contribution pour distinguer le Nord-du-Québec et le Saguenay-Lac-Saint-Jean, à partir de 1987. Par ailleurs, l'évolution historique des frontières des régions administratives a principalement visé à se rapprocher des espaces identitaires de même qu'à obtenir des populations totales semblables.

\section{Les régions administratives québécoises ne sont pas établies sur la base d'attributs naturels, sauf la Gaspésie, qui forme une péninsule, de même que Laval et Montréal qui reposent sur des îles.}

Tableau 1 - Population, densités et superficies des régions administratives

\begin{tabular}{|c|c|c|c|c|c|c|}
\hline No & Région administrative & $\begin{array}{c}\text { Population } \\
2006 \\
\end{array}$ & $\begin{array}{c}\text { Population } \\
2014 \\
\end{array}$ & $\begin{array}{c}\text { Variation } \\
2006-14 \\
\end{array}$ & $\begin{array}{c}\text { Densité } \\
2014 \\
\end{array}$ & $\begin{array}{c}\text { Superficie } \\
\mathbf{k m}^{2} \\
\end{array}$ \\
\hline 01 & Bas-Saint-Laurent & 201600 & 200292 & -1308 & 9 & 22185 \\
\hline 02 & Saguenay-Lac-Saint-Jean & 274286 & 277786 & 3500 & 3 & 95893 \\
\hline 03 & Capitale-Nationale & 668948 & 731838 & 62890 & 39 & 18639 \\
\hline 04 & Mauricie & 260407 & 266794 & 6387 & 8 & 35452 \\
\hline 05 & Estrie & 301058 & 320008 & 18950 & 31 & 10195 \\
\hline 06 & Montréal & 1872136 & 1988243 & 116107 & 3991 & 498 \\
\hline 07 & Outaouais & 345027 & 383182 & 38155 & 13 & 30504 \\
\hline 08 & Abitibi-Témiscamingue & 144887 & 147868 & 2981 & 3 & 57340 \\
\hline 09 & Côte-Nord & 96569 & 94906 & -1663 & 0 & 236700 \\
\hline 10 & Nord-du-Québec & 40291 & 44256 & 3965 & 0 & 718229 \\
\hline 11 & Gaspésie-Îles-de-Madeleine & 95206 & 92472 & -2734 & 5 & 20272 \\
\hline 12 & Chaudière-Appalaches & 397133 & 419755 & 22622 & 28 & 15071 \\
\hline 13 & Laval & 372495 & 420870 & 48375 & 1711 & 246 \\
\hline 14 & Lanaudière & 433901 & 492234 & 58333 & 40 & 12313 \\
\hline 15 & Laurentides & 518664 & 586051 & 67387 & 29 & 20560 \\
\hline 16 & Montérégie & 1383294 & 1508127 & 124833 & 136 & 11111 \\
\hline 17 & Centre-du-Québec & 225971 & 239990 & 14019 & 35 & 6921 \\
\hline- & Superficies terrestres & - & - & - & - & $1312124^{51}$ \\
\hline- & Superficies lacustres & - & - & - & - & 201755 \\
\hline- & Superficies marines & - & - & - & - & 153562 \\
\hline- & Total - Le Québec & 7631873 & 8214672 & 582799 & $6,3^{52}$ & 1667441 \\
\hline
\end{tabular}

(Source : Institut de la statistique du Québec) 
Les régions administratives du Québec sont nombreuses et fort différentes. Certaines sont surdimensionnées, agglomérant plusieurs régions culturelles, alors que d'autres sont très petites et ne constituent qu'une composante de la dynamique de la grande région métropolitaine de Montréal. D'ailleurs, cette région métropolitaine empiète sur plusieurs MRC et régions administratives. Néanmoins, on peut constater que plusieurs régions affichent une taille semblable
(10 régions sur 17 possèdent une superficie variant de 10000 à $50000 \mathrm{~km}^{2}$ ). Rappelons que ces entités territoriales ne possèdent pas d'instance politique, à proprement parler. Malgré tout, le gouvernement provincial a longtemps tenté de coordonner ses actions sur une base régionale par l'intermédiaire des Conseils régionaux de développement (CRD) et des Conférences régionales des élus (CRÉ) ${ }^{53}$.

\section{Carte 2 - Les régions physiographiques et administratives du Québec ${ }^{54}$}

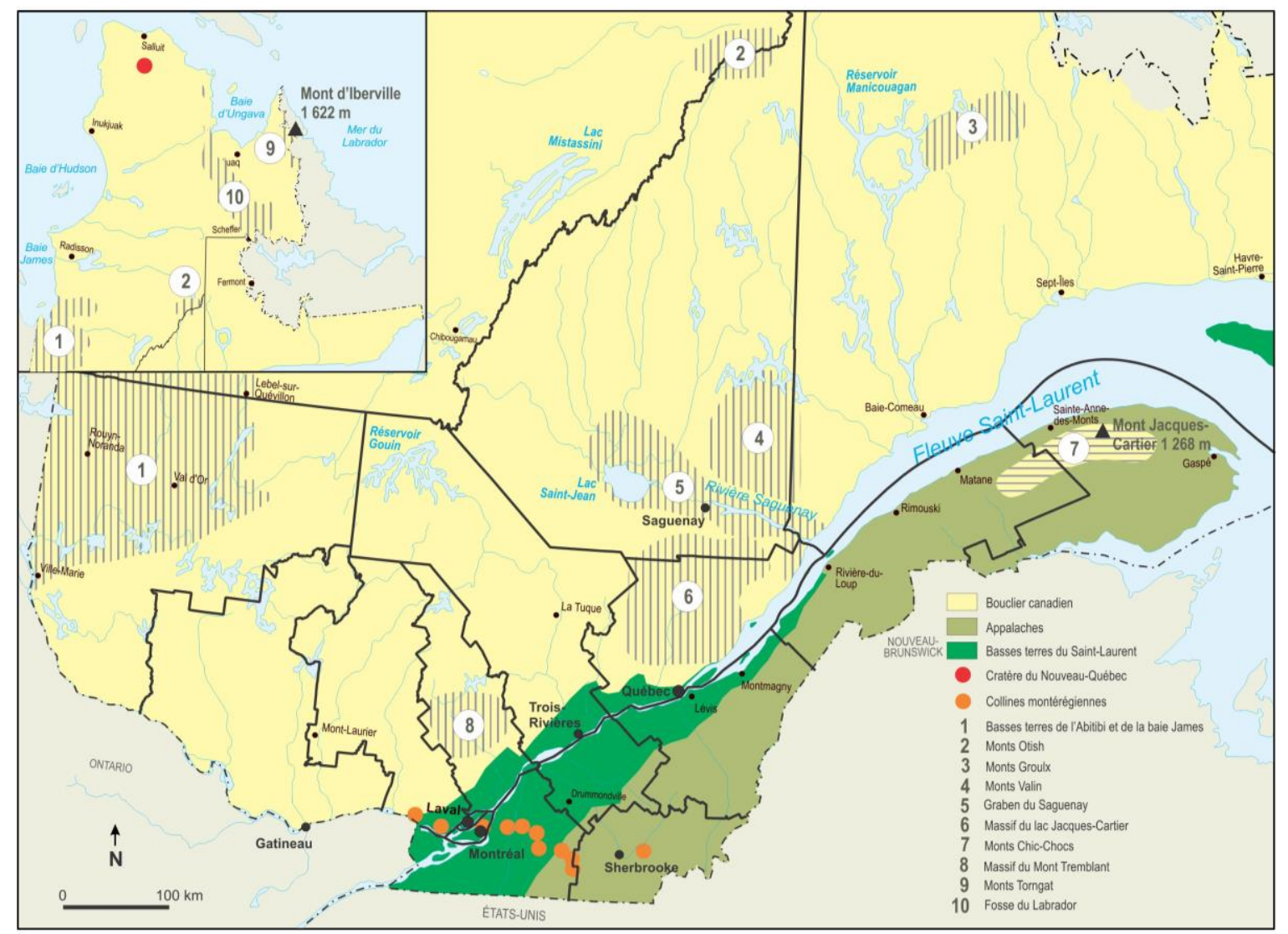

Fonds de carte: Ministere des Ressources naturelles et de la Faune

Au-delà de leurs spécificités respectives, les régions administratives du Québec peuvent être regroupées par catégories. Ainsi, on oppose aisément les régions centrales, ayant des assises territoriales dans la plaine du Saint-Laurent, des régions périphériques. En réalité, plusieurs régions administratives comportent à la fois des espaces centraux, situés dans la plaine du Saint-Laurent, et des espaces ruraux et forestiers s'apparentant au contexte des régions périphériques (Laurentides, Lanaudière, Mauricie, Capitale-Nationale et Bas Saint-Laurent). Qualifiées par certains de croissant péri-nordique ${ }^{55}$, les régions périphériques comprennent l'Abitibi-Témiscamingue, le SaguenayLac-Saint-Jean, la Côte-Nord, le Nord-du-Québec le Bas-Saint-Laurent, la Gaspésie-Îles-de-laMadeleine. Celles-ci disposent d'une économie axée sur les ressources naturelles (mine, forêt, pêche, hydroélectricité, etc.) et des paysages au relief ondulé ou accidenté. Malgré leur peuplement plus tardif que dans l'axe laurentien, ces territoires jouissent d'un fort sentiment d'appartenance. Ce sentiment peut mener à des revendications sociales, économiques ou politiques. 
$\mathrm{Au}$ Québec, les revendications régionalistes interpellent les gouvernements provincial et, incidemment, fédéral pour la qualité de services publics et l'utilisation des ressources naturelles. Avec seulement 10,4\% de la population du Québec, un solde migratoire négatif et des taux de chômage généralement plus élevés que la moyenne, ces milieux se considèrent laissés pour compte, à divers degrés ${ }^{56}$. Aux yeux de plusieurs, les régions périphériques sont victimes des lois implacables de l'échange inégal et des stratégies centralisatrices des gouvernants ${ }^{57}$. Des demandes de décentralisation des pouvoirs s'appuient sur cette injustice territoriale réelle ou perçue. Les régions périphériques et les municipalités qui les composent défendent leur droit d'exister et de jouir de conditions de vie équitables à travers le concept d'occupation du territoire ${ }^{58}$.

Un deuxième palier de structures régionales existe depuis 1979, au Québec. Il s'agit des
Municipalités régionales de comté (MRC). Ces organismes micro-régionaux ou supra-locaux sont des structures de type municipal ou communal œuvrant dans les champs de l'évaluation foncière, de l'enfouissement sanitaire, de l'aménagement du territoire et, depuis peu, du développement régional. Étant au nombre de 103, soit 87 MRC et seize territoires équivalents ${ }^{59}$ et affichant une superficie moyenne de $12739 \mathrm{~km}^{2}$, elles sont plus proches des citoyens que les régions administratives (voir le tableau 2). En effet, ces MRC ont été façonnées selon le principe des territoires d'appartenance par opposition aux régions administratives, d'échelle supérieure, qui ont été érigées, du moins au départ, pour couvrir les espaces économiques polarisés par les villes moyennes éparpillées sur l'espace habité ${ }^{60}$. Les frontières administratives de MRC sont le plus souvent géométriques car elles s'adaptent aux frontières des municipalités locales qui les subdivisent.

Tableau 2 - Populations, superficies et densités moyennes des unités régionales au Québec

\begin{tabular}{|l|c|c|c|c|c|}
\hline \multicolumn{1}{|c|}{ Type de régions } & $\begin{array}{c}\text { Nombre } \\
\text { d'unités }\end{array}$ & $\begin{array}{c}\text { Population } \\
\text { moyenne }\end{array}$ & $\begin{array}{c}\text { Superficie } \\
\text { moyenne }\end{array}$ & $\begin{array}{c}\text { Densité } \\
\text { moyenne }\end{array}$ & $\begin{array}{c}\text { Corrélation } \\
\text { Pop./sup. }\end{array}$ \\
\hline Régions administratives & 17 & 483216 & 77184 & 357,6 & $-0,32$ \\
\hline MRC ou terr. équivalents & 103 & 78987 & 12938 & 156,6 & $-0,07$ \\
\hline
\end{tabular}

(Source : Institut de la statistique du Québec)

Contrairement aux régions administratives, les MRC sont gérées par des élus locaux « au second degré », c'est-à-dire les maires des municipalités locales dont le territoire est inclus à l'intérieur des frontières de la MRC ${ }^{61}$. Ceux-ci nomment un «maire» de MRC que l'on qualifie de préfet. Cependant, quelques préfets de MRC sont maintenant élus directement par la population régionale, une innovation sociale qui pourrait faire boule de neige. Il y a actuellement quatorze préfets de MRC d'élus au suffrage universel ${ }^{62}$. Le dynamisme des MRC diffère énormément selon le contexte (municipalités membres étant rurales ou mixtes, proximité ou éloignement par rapport aux grands pôles urbains, etc.) et la volonté politique des représentants municipaux en fonction ${ }^{63}$. C'est que les élus locaux siégeant aux conseils de MRC paraissent se considérer d'abord redevables à leur municipalité locale et ils s'entendent souvent, de manière plus ou moins tacite, pour réduire l'action de la MRC à sa plus simple expression.
Les MRC se sont vues attribuées des responsabilités supplémentaires par le gouvernement du Québec au fil des ans, par exemple la gestion des cours d'eau et la couverture des risques d'incendies à travers l'élaboration d'un schéma. Ces délégations plutôt techniques, sans financement additionnel, ne sont pas vraiment considérées comme de la décentralisation. En outre, les MRC situées à la périphérie de l'écoumène, le plus souvent au sein des régions périphériques, ont des pouvoirs supplémentaires par rapport aux terres publiques et la forêt, car leur territoire contient des espaces inhabités, généralement sous couvert forestier. D'autre part, avec la réforme municipale de 2001 au Québec, plusieurs villes regroupées ou «agglomérations » ont également obtenu des compétences de MRC. Celles-ci sont ainsi exclues des MRC voisines et ces dernières cessent d'être des lieux de concertation entre les acteurs des mondes urbain et rural. Toutefois, ces villes-MRC peuvent elles-mêmes contenir des espaces agricoles, mais cette 
unification politique risque plutôt de favoriser l'urbanisation de la campagne péri-urbaine. D'autre part, la création des Communautés métropolitaines en 2006, structures qui opèrent à l'échelle des régions métropolitaines de recensement $(\mathrm{RMR})^{64}$, vient court-circuiter et rendre potentiellement caduques les MRC dont le territoire est totalement ou partiellement inclus dans la communauté métropolitaine de Montréal (CMM) ou celle de Québec $(\mathrm{CMQ})^{65}$.

\section{LA RÉFORME ACTUELLE DE LA GOUVERNANCE RÉGIONALE}

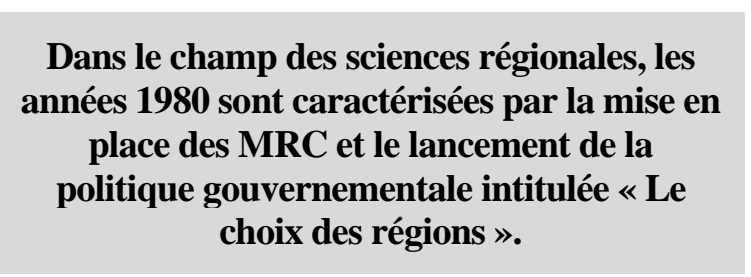

Les politiques québécoises en matière de développement régional ont toujours oscillées entre l'échelle macro-régionale et l'échelle microrégionale. Conséquemment, la région administrative et la MRC sont interpellées à tour de rôle dans l'histoire récente de la province afin de constituer le pivot des politiques territoriales. Cette valse-hésitation n'est pas sans causer de problème. Revenons brièvement sur les grands moments des stratégies québécoises de gouvernance territoriale et de développement régional.

Quelques années après la création des régions administratives, le gouvernement du Québec instaure l'Office de planification et de développement du Québec (OPDQ). Cet organisme, aboli en 1991, œuvre à coordonner les actions gouvernementales en fonction des régions administratives. Nous sommes à l'époque des fortes ambitions gouvernementales dans le champ du développement régional. Partout en Occident, les gouvernements nationaux ou provinciaux tentent de modeler les territoires à l'aide de politiques généralement inspirées par la théorie des pôles de croissance et de développement ${ }^{66}$. Au Québec, la sphère des idées est marquée par le rapport de Higgins, Martin et Raynauld (1971) sur «Les orientations du développement économique régional dans la province de Québec ${ }^{67}$. Tirant son origine des écrits des économistes François Perroux et Jacques Boudeville, la vision de plusieurs est la suivante : les investissements publics devraient se concentrer en zones urbaines ou métropolitaines, seuls territoires capables de créer de la richesse et de la transmettre aux différents paliers du système urbain ainsi qu'aux espaces ruraux que les entourent. Influencé en partie par cette lecture,
l'OPDQ s'intéresse prioritairement aux armatures urbaines et aux filières industrielles dans ses plans d'aménagement et de développement des années 1970.

Dans le champ des sciences régionales, les années 1980 sont caractérisées par la mise en place des MRC et le lancement de la politique gouvernementale intitulée «Le choix des régions » (1983). Celle-ci est au diapason de la tendance sociétale envers le développement endogène ou local. En attendant de trouver la voie d'un arrimage entre aménagement et développement à l'échelle mercéenne $^{68}$, de grands processus macro-régionaux sont encore à l'œuvre. Ainsi, la tenue de sommets économiques régionaux accapare beaucoup d'énergie dans les officines gouvernementales régionales $^{69}$. Dans la décennie suivante, on maintient ces grands processus de concertation en les simplifiant selon le concept de planification stratégique quinquennale issue de la stratégie nommée « Développer les régions du Québec » (1991). Cette planification est orchestrée par les Centres régionaux de développement (CRD) qui remplacent, en quelque sorte, l'OPDQ. Les Centres locaux de développement (CLD) voient également le jour à la suite du lancement de la « Politique de soutien au développement local et régional » (1997). Cette nouvelle structure fonctionnera au palier microrégional en utilisant les territoires de MRC.

Les politiciens n'étant jamais à court d'idées, les années 2000 verront naître les Conférences régionales des élus (CRÉ). Ces forums dirigés par une assemblée régionale d'élus municipaux viennent prendre la relève des CRD en 2003. La période qui suit est aussi marquée par la « Politique nationale de la ruralité » (2002), le «Plan d'action gouvernemental à l'intention des municipalités dévitalisées » (2006) et la « Stratégie pour assurer l'occupation et la vitalité des territoires » (2011). Ces politiques fortes en verbe procurent des financements aux MRC et municipalités locales sans imposer trop de contraintes administratives. 
Ces dernières vont fréquemment embaucher des agents de développement rural pour administrer ces fonds et animer les milieux locaux. Ce système à deux paliers régionaux subsiste jusqu'aux réformes gouvernementales de 2015. Il faut aussi noter la présence du gouvernement fédéral en matière de développement régional : il s'agit d'interventions ponctuelles, notamment par le biais de l'agence de développement économique du Canada pour les régions du Québec (DEC Canada), ou d'actions plus soutenues par l'intermédiaire des Sociétés d'aide au développement des collectivités (SADC) ou des Centres d'aide aux entreprises (CAE).

La réforme de 2015 se réalise dans un contexte d'austérité budgétaire. Le gouvernement libéral de Philippe Couillard procède ainsi à la mise au rancart des CRÉ dans un texte législatif adopté le 21 avril. Les engagements des CRÉ, notamment les ententes spécifiques de régionalisation, sont abandonnées et ceux-ci se départissent de leur personnel et de leurs immeubles. Les CLD subissent également une

\section{CONCLUSION}

Le Québec compte donc deux paliers de territoires régionaux, les régions administratives et les MRC. En plus des divers problèmes de découpage des frontières précédemment discutés, ces régions, et les organismes qui les gèrent, disposent de relativement peu de pouvoirs. Les régions administratives demeurent des lieux de déconcentration des fonctionnaires et d'organisation des quelques services et interventions sur une base macrorégionale. L'instrument de coordination que constituaient les CRÉ est maintenant dissous, à la suite d'une réforme inspirée par le régime d'austérité budgétaire. Pour leur part, les MRC se limitent à être des organismes de type municipal ayant assez peu de financement et de légitimité politique, sauf quelques-unes qui profitent de certaines redevances sur les ressources de leur Territoires non-organisés (TNO). D'ailleurs, la constitution de villes ayant certaines compétences de MRC a confirmé la périphérisation des espaces ruraux, nonobstant les beaux discours de la politique nationale de la ruralité.

Sur la durée, on remarque une valse-hésitation constante du gouvernement québécois dans le importante diminution de leur financement. Les MRC seront responsables d'assurer ou non la poursuite des activités d'accompagnement de l'entrepreneuriat local, par exemple en intégrant à leur structure un service de développement économique. Même si les CRÉ n'étaient pas parfaites, en particulier quant à la composition de leurs conseils d'administration, leur démantèlement laisse un vide important. En effet, au cours des cinq dernières décennies, il y a toujours eu une structure de coordination à l'échelle des régions administratives au Québec, sorte d'embryon d'un gouvernement régional. Cela ne sera plus le cas. Cependant, il est question de mettre en place des tables régionales des préfets de MRC. Cette idée apparait paradoxale, ramener le développement régional à l'échelle des MRC pour ensuite leur demander de se coordonner à l'échelle macrorégionale. De plus, le nombre et la diversité des intervenants régionaux en situation de décision seront fortement réduits, si l'on compare ces futures tables des préfets aux anciennes CRÉ.

choix d'un palier régional prioritaire. Trop grandes, dans le cas des régions administratives, ou trop petites, dans le cas des MRC, les structures existantes ne semblent pas adaptées à la réalité socioterritoriale du Québec qui contiendrait environ 25 régions historiques ou culturelles ${ }^{70}$. Au surplus, la pratique des ministères québécois d'utiliser un cadre territorial différent de celui des régions administratives reste bien vivante, 50 ans après leur cartographie, spécialement en matière de tourisme et de santé. Si l'on ajoute à cela la mise en place d'organismes de bassins-versants à l'échelle micro-régionale sans tenir compte des territoires de MRC et le Nord tel que délimité dans le Plan Nord $^{71}$, on constate aisément que l'hétérogénéité spatiale et institutionnelle continue de complexifier l'organisation politico-territoriale du Québec ${ }^{72}$.

Trop grandes, dans le cas des régions administratives, ou trop petites, dans le cas des MRC, les structures existantes ne semblent pas adaptées à la réalité socioterritoriale du Québec qui contiendrait environ 25 régions historiques ou culturelles. 
$\mathrm{Au}$ moment où plusieurs maires des super villesMRC, comme Saguenay ou Trois-Rivières, dominent leur scène régionale respective, il est permis de se demander si des gouvernements régionaux forts ne constitueraient pas un recul. En effet, l'état central québécois est en meilleure posture pour assurer des démarches de planification et de redistribution. De plus, des instances décentralisées dotées de pouvoirs réels et de représentants élus seraient des petits milieux susceptibles de laisser place à la collusion ou de devenir des fiefs de groupes coalisés fonctionnant de manière plus ou moins démocratique. À cet égard, le cas de Laval, au cœur même d'un espace métropolitain, est lourd d'enseignements. Au demeurant, la situation de demi-gouvernement de la province fédérée du Québec laisse entrevoir peu de délégations de compétences à l'interne ${ }^{73}$, en vertu de la règle d'auto-préservation qui semble exister au sein des structures politiques à toutes les échelles.

À tort ou à raison, le gouvernement du Québec paraît plus préoccupé par les questions des frontières «nationales » et du développement nordique. En effet, au moment d'écrire ces lignes, ce gouvernement réclame d'Ottawa un amendement constitutionnel pour intégrer officiellement les eaux territoriales au territoire de la province, au pourtour de la région administrative du Nord-duQuébec. Cette demande vise à corriger une « erreur historique » réalisée lorsque le gouvernement fédéral a cédé le territoire de l'Ungava au Québec, en $1912^{74}$. La question du «Labrador perdu» demeure également un élément important de l'imaginaire québécois même si très peu de Québécois y ont un jour mis les pieds. La région s'incline alors devant l'État central quand il est question de frontières, terrestres ou maritimes. Cela confirme le statut subordonné que conservera toujours le palier régional dans tous les Étatsnations, voire au sein d'États fédérés : un territoire de seconde classe ouvert aux manipulations politiques de toutes sortes, un mal nécessaire soumis au regard attentif des maîtres du gouvernement central. Néanmoins, son pouvoir et son utilité proviennent de la correspondance des frontières avec un substrat social cohérent et solidaire, fondement de revendications égalitaristes. C'est cela qui peut faire la différence entre des corps sans âmes et des unités sociospatiales participant aux inévitables rapports de force sociopolitiques et économiques qui traversent et façonnent la société.

\section{BIBLIOGRAPHIE ET NOTES}

${ }^{1}$ Martin Simard est géographe et urbaniste. Il œuvre comme professeur de géographie urbaine et régionale à l'Université du Québec à Chicoutimi depuis 2002 en plus d'être membre régulier du Centre de recherche sur le développement territorial.

${ }^{2}$ Claval, P. (2011). Histoire de la géographie, Paris, Presses universitaires de France, 126 pages.

${ }^{3}$ Bailly, A. S. et Ferras, R. (2010). Éléments d'épistémologie de la géographie, Paris, Armand Colin, 191 pages.

${ }^{4}$ Nos travaux cadrent avec cette dernière approche.

${ }^{5} \mathrm{Le}$ Monde (2014). La carte à treize régions définitivement adoptée, édition du 17 décembre 2014 [en ligne] http://www.lemonde.fr/politique/article/2014/12/ 17/la-carte-a-13-regions-definitivement adoptee 4542278_823448.html\#1RU0kuSCYE5IH7hX.99

${ }^{6}$ Morissonneau, C. (2015). « La région comme rempart contre l'homogénéisation du monde », dans Mukakayumba, É. et Lamarre, J. (dir.) La géographie en action. Une collaboration entre la science et le politique, Québec, Presses de l'Université du Québec, p.41-54.

${ }^{7}$ OAP (2012). L'état québécois en perspective. Les institutions objets de décentralisation politique, Observatoire de l'administration publique, École nationale d'administration publique, 15 pages [en ligne] http://cerberus.enap.ca/Observatoire/docs/Etat_quebeco is/a-territorial.pdf, consulté le 16 octobre 2015.

${ }^{8}$ Alexandre, F. et Genin, A. (2008). «Introduction », dans Alexandre, F. et Génin, A. (dir.) Continu et discontinu dans l'espace géographique, Tours, Presses universitaires François-Rabelais, p.11-74.

${ }^{9}$ Bailly A.S. (1996). « La frontière : des coquilles de l'homme au maillage du système monde », Le Globe. Revue genevoise de géographie, vol. 136, no 136, p. 17-23.

${ }^{10}$ Morin, R. (2006). La régionalisation au Québec. Les mécanismes de développement et de gestion des territoires régionaux et locaux (1960-2006), Montréal, Éditions Saint-Martin, 75 pages.

${ }^{11}$ Bailly, A.S. et Beguin, H. (2003). Introduction à la géographie humaine, Paris, Armand Colin, 192 pages.

${ }^{12}$ Dorion, H. (2007). « Un territoire ou des territoires », dans De Koninck, M.C. Le Québec. Territoires : habitat, ressources et imaginaire, Québec, Multimondes et Musée de la civilisation, p.9-19.

${ }^{13}$ Raffestin, C. (1980). Pour une géographie du pouvoir, Paris, Masson, 130 pages.

${ }^{14}$ Brunet, R. (1995) « La géographie, science des territoires et des réseaux », Cahiers de géographie $d u$ Québec, vol. 39, no 108, p. 477-482. 
${ }^{15} \mathrm{Du}$ moins, dans l'acceptation générale du sens de territoire. Cependant, dans le champ de la géopolitique, le territoire peut être vu comme l'espace soumis à l'autorité d'un État.

${ }^{16}$ Baud, P., Bourgeat, S. et Bras, C. (2013). Dictionnaire de géographie ( $5^{\mathrm{e}}$ éd.), Paris, Hatier, 608 pages.

${ }^{17}$ Petit, T. (1990). Satrapes et satrapies dans l'empire achéménide de Cyrus le Grand à Xerxès Ier, Paris, Éditions les belles lettres, 274 pages.

${ }^{18}$ Bérubé, P. (2000). « La restructuration interne des États : faits et tendances dans les pays de l'OCDE », Télescope, vol. 7, no 1, 9 pages.

${ }^{19} \mathrm{~L}$ 'expression régionalisation peut également faire référence à l'organisation spatiale différenciée d'un phénomène.

${ }^{20}$ Gourdin, P. (2015). Manuel de géopolitique, éd. Diploweb.com [en ligne] http://www. diploweb.com/8Les-marques-du-decoupage.html

${ }^{21}$ Belhedi, A. (2013). La dimension géo-stratégique de la région, Communication au Colloque International «Quelle régionalisation dans les pays du Maghreb?», Tunis, 9 décembre 2013, [en ligne] https://amorbelhedi.wordpress.com/2013/12/31/ladimension-geo-strategique-de-la-region/.

${ }^{22}$ Faludi, A. (2014). " Place is a no-man's land », Geographia Polonica, vol. 88, no 1, p. 5-20.

${ }^{23}$ Pour en savoir plus sur le débat entre «continuistes » et « discontinuistes », voir Alexandre et Génin (2008) ou Gay (2004).

${ }^{24}$ Di Méo, G. (1998). Géographie sociale et territoires, Paris, Nathan, 320 pages.

${ }^{25}$ Dorion, H. et Lacase, J.-P. (2011). Le Québec, territoire incertain, Québec, Septentrion, 328 pages.

${ }^{26}$ Raffestin, C. (1980). Pour une géographie du pouvoir, Paris, Masson, 130 pages.

${ }^{27}$ Girard, N. (2004). « La région : une notion géographique », Ethnologie française, vol. 34, no 1, p. 107-112. ${ }^{28}$ Hamelin, L.-E. (1994). Le rang d'habitat. Le réel et l'imaginaire, Montréal, Hurtubise, 332 pages.

${ }^{29}$ Polèse, M., Shearmur, R. et Terral, L. (2015). Économie urbaine et régionale : Introduction à la géographie économique (4 éd.), Paris, Économica, 416 pages.

${ }^{30}$ Sassen, S. (2009). La globalisation, une sociologie, Paris, Gallimard, 341 pages.

${ }^{31}$ Hertzog, A. et Sierra, A. (2010). « Penser la ville et l'urbain, les paradoxes de la géographie française », EchoGéo [En ligne] http://echogeo.revues. org/11898, consulté le 14 octobre 2015.

${ }^{32}$ Grafmeyer, Y. et Joseph, I. (2009). L'école de Chicago: naissance de l'écologie urbaine, Paris, Flammarion, 377 pages.

${ }^{33}$ Jean, B. (2006). «Le développement territorial : un nouveau regard sur les régions du Québec », Recherches sociographiques, vol. 47, $\mathrm{n}^{\circ} 3$, p. 465-474.

${ }^{34}$ Favreau, L., Robitaille, M. et Tremblay, D. dir. (2002). Quel avenir pour les régions, Chaire de recherche du
Canada en développement des collectivités, Université du Québec en Outaouais, Gatineau, 343 pages.

${ }^{35}$ Coulangeon, P. (2004). «Classes sociales, pratiques culturelles et styles de vie : le modèle de la distinction est-il obsolète ? », Sociologie et sociétés, vol. 36, $\mathrm{n}^{\circ} 1$, p. 59-85.

${ }^{36}$ Beaudry, R. et Dionne, H. (1994). " Rechercheintervention en communauté villageoise ", Nouvelles pratiques sociales, vol. $7, \mathrm{n}^{\circ} 2$, p. 141-152.

${ }^{37}$ Simard, M. (2000). « Les communautés locales et l'espace monde. Les processus identitaires de la postmodernité », Géographie et cultures, no 36, p. 3-21. ${ }^{38}$ Laurin, S., Klein, J.-L. et Tardif, C. (2001). «Géographie et société. Vers une géographie citoyenne », dans Laurin, S., Klein, J.-L. et Tardif, C. (dir.) L'éducation géographique. Formation du citoyen et conscience territoriale, Québec, Presse de l'Université du Québec, p. 1-12.

${ }^{39}$ L'immigration québécoise s'est diversifiée après la Deuxième Guerre mondiale, ce qui contribue à la présence de nombreuses communautés culturelles dans la province, principalement à Montréal et dans les régions limitrophes.

${ }^{40}$ Courville, S. (2000). Le Québec. Genèse et mutations du territoire, Québec, Presses de l'Université Laval / Les éditions de l'IQRC, 508 pages.

${ }^{41}$ Manzagol, C. et Sénécal, G. (1996). « La région géographique », dans Proulx, M.-U. (dir.) Le phénomène régional au Québec, Québec, PUQ, p. 93-112.

${ }^{42} \mathrm{~L}$ 'écoumène est évalué ici à $180000 \mathrm{~km}^{2}$.

${ }^{43}$ Blanchard, R. (1960). Le Canada français, Montréal, Librairie Artheme Fayard, 314 pages.

${ }^{44}$ Certains auteurs indiquent plutôt l'année 1967 comme moment de création des régions administratives, notamment Manzagol et Sénécal (1996).

${ }^{45}$ Dugas, C. (1986). « Région et régionalisation au Québec depuis Raoul Blanchard », Cahiers de géographie du Québec, vol. 30, no 80, p. 189-202 ET Manzagol, C. et Sénécal, G. (1996). « La région géographique », dans Proulx, M.-U. (dir.) Le phénomène régional au Québec, Québec, PUQ, p. 93-112.

${ }^{46}$ Proulx, M.-U. (2008). « 40 ans de planification territoriale au Québec », dans Gauthier, M., Gariépy, M. et Trépanier M.-O., Renouveler l'aménagement et l'urbanisme. Planification territoriale, débat public et développement durable, Montréal, Presses de l'Université de Montréal, p. 23-54.

${ }^{47}$ Matthews Glenne, J. (1986). « La décentralisation de l'aménagement du territoire : mythe ou réalité? », Les Cahiers de droit, vol. 27, $\mathrm{n}^{\circ}$ 2, p. 355-370.

${ }^{48}$ Les noms de régions qui sont associés aux numéros sur la carte peuvent être vus dans le tableau 1 .

${ }^{49}$ Les $49^{\mathrm{e}}$ et $55^{\mathrm{e}}$ parallèles nord sont employés pour délimiter le Nord-du-Québec par rapport à l'AbitibiTémiscamingue et la Côte-Nord. Le $48^{\mathrm{e}}$ parallèle sépare le Saguenay-Lac-Saint-Jean de la région de la Capitale- 
nationale. De plus, le $75^{\mathrm{e}}$ méridien ouest est utilisé pour distinguer le Saguenay-Lac-Saint-Jean de la Côte-Nord. Finalement, l'usage du $52^{\mathrm{e}}$ parallèle pour délimiter le Labrador terre-neuvien et de la Côte-Nord québécoise fait l'objet d'un contentieux territorial interprovincial depuis 1927.

${ }^{50}$ Lefebvre, C. (2011). « La décolonisation d'un lieu commun. L'artificialité des frontières africaines : un legs intellectuel colonial devenu étendard de l'anticolonialisme "), Revue d'Histoire des sciences humaines, vol. 1, $\mathrm{n}^{\circ}$ 24, p. 77-104.

${ }^{51}$ Certaines sources, notamment Statistique Canada, évaluent les superficies terrestres du Québec à 1 $365128 \mathrm{~km}^{2}$ pour total de $1542056 \mathrm{~km}^{2}$ (superficies terrestres et lacustres).

${ }^{52}$ Le calcul de densité s'applique sur les superficies terrestres de la province, soit $1312124 \mathrm{~km}^{2}$.

${ }^{53}$ Ces organismes n'existent plus. Le gouvernement du Québec travaille présentement à organiser des tables régionales de préfets de MRC pour assumer certaines tâches anciennement dévolues aux CRÉ.

${ }^{54}$ L'auteur remercie M. Carl Brisson, professionnel de recherche au laboratoire LERGA de l'UQAC, pour la conception des cartes 2, 3, 4 et 5 .

${ }^{55}$ Leblanc, P., Girard, C., Côté, S. et Potvin, D. (2003). «La migration des jeunes et le développement régional dans le croissant péri-nordique du Québec », Recherches sociographiques, vol. 44, no 1, p. 35-55.

${ }^{56}$ Dugas, C. (1986). « Région et régionalisation au Québec depuis Raoul Blanchard », Cahiers de géographie du Québec, vol. 30, no 80, p. 189-202.

${ }^{57}$ Fournis, Y. (2012). « Penser la ruralité et son développement au GRIDEQ entre 1970 et 2000 : du mouvement social localisé à la construction symbolique des communautés territoriales », Cahiers de géographie du Québec, vol. 56, n 157, p. 153-172.

${ }^{58}$ Bouchard, R. (2013). Y a-t-il un avenir pour les régions? Un projet d'occupation du territoire, Montréal, Écosociété, 270 pages.

${ }^{59}$ Le chiffre 103 est obtenu en additionnant les $87 \mathrm{MRC}$, les quatorze villes ou agglomérations ayant certaines compétences de MRC ainsi que les territoires de l'Eeyou-Istchee-Baie-James et du Nunavik.

${ }^{60}$ Proulx, M.-U. (1992). « Appartenance, utilités, fonctions. Le cas des milieux MRC du Québec », Revue canadienne des sciences régionales, vol. $15, \mathrm{n}^{\circ} .2$, p. 307-325.

${ }^{61}$ Quesnels, L. (1986). « La démocratie municipale au Québec », Politique, no 9, p. 61-97.

${ }^{62}$ MAMOT (2014). L'organisation municipale et régionale au Québec en 2014, Ministère des affaires municipales et de l'occupation du territoire, 21 pages [en ligne] http://www.mamrot.gouv.qc.ca/pub/organisation _municipale/organisation_territoriale/organisation_mun icipale.pdf, consulté le 14 octobre 2015.
${ }^{63}$ Gravel, R.-J. (1999). Les institutions administratives locales et régionales au Québec. Structures et fonctions, Québec, Presses de l'Université du Québec, 131 pages. ${ }^{64}$ La région métropolitaine de recensement (RMR) est un découpage territorial de Statistique Canada qui permet de délimiter l'aire de navettage d'une ville de plus de 100000 habitants. Ce terme n'implique généralement aucun statut juridique particulier et sert strictement à recueillir des statistiques démographiques et socioéconomiques. Néanmoins, le gouvernement du Québec a fondé des organismes administratifs nommés « communautés métropolitaines » à Montréal et à Québec, organismes ayant une base territoriale équivalente aux territoires de RMR.

${ }^{65} \mathrm{Ces}$ MRC sont les suivantes, pour la CMM : Beauharnois-Salaberry, Deux-Montagnes, L'Assomption, Vallée-du-Richelieu, Rouville et VaudreuilSoulanges. Ces MRC sont les suivantes, pour la CMQ : Côte-de-Beaupré et Jacques-Cartier.

${ }^{66}$ Couzon, I. (2003). « Les espaces économiques de François Perroux (1950). Organisation de l'espace et aménagement du territoire dans l'économie et la géographie françaises au milieu du $\mathrm{XX}^{\mathrm{e}}$ siècle », Revue d'Histoire des sciences humaines, vol. 2, n 9, p. 81-102. ${ }^{67}$ Polèse, M. et Shearmur, R. (2003). « R.I.P.- H.M.R : À propos du concept de pôle de développement et des stratégies de développement économique des régions québécoises », Revue Canadienne de science régionales, vol. 24, no 1, p .61-86.

${ }^{68}$ Klein, J.-L. (2010). « Changements de paradigme en géographie et aménagement du territoire », Cahiers de géographie du Québec, vol. 54, n 151, p. 133-152.

${ }^{69}$ Bergeron, G. (1986). La contribution du Sommet sur le développement et l'économie de la région 02 à la mise en place d'un processus d'auto-développement régional, Chicoutimi, Université du Québec à Chicoutimi, 42 pages.

${ }^{70}$ Cette évaluation provient de Racine, J.-B. et Villeneuve, P. (1992). Canada, Collection Géographie Universelle, Paris, Hachette/Reclus.

${ }^{71}$ Le Plan Nord du gouvernement du Québec définit ce territoire comme tout ce qui est au nord du $49^{\mathrm{e}}$ parallèle, soit un espace qui contient l'ensemble de la région administrative du Nord-du-Québec mais aussi de larges segments du Saguenay-Lac-Saint-Jean et de la CôteNord.

${ }^{72}$ Ce constat a été fait par Pierre Bérubé dès 1993.

${ }^{73} \mathrm{Ce}$ lien entre, d'une part, la situation constitutionnelle et, d'autre part, la politique de régionalisation au Québec a été établit par Lionel Robert en 1991.

${ }^{74}$ Pour plus de détails à ce sujet, voir le livre de H. Dorion et J.-P. Lacasse (2011) référencé en bibliographie. 


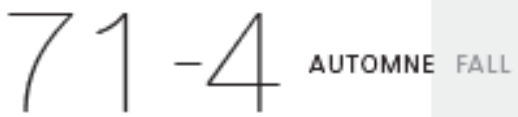

REVUE TRIMESTRIELLE

RELATIONS INDUSTRIELLES

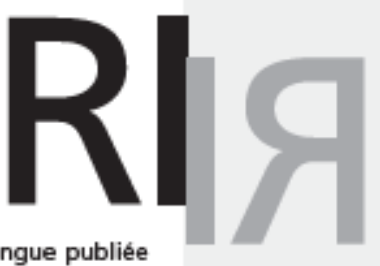

Revue trimestrielle bilingue publiée depuis 1945 par le Département des relations industrielles de l'Université Laval

ARTICLES

Multi-Scalar Trade Unionism:

Lessons from Maritime Unions

PETER FAIRBROTHER AND VCTOR OYARO GEKARA

Le système d'emploi des travailleurs agricoles saisonniers: topographie d'un rapport salarial multipartite DALA GESUALDI-FECTEAIJ

What's Next after Psychological Contract Violation?

SYLVE GUERRERO AND MICKAEL NAULLLEAN

Le rôle médiateur de l'engagement au travail dans la relation entre la justice organisationnelle et les comportements de citoyenneté:

le cas de la police française

MATHIEU MOUNES ET AMAR FALL.

Analyzing Exclusion in Global Worker Health Policy

JEFREY HLGERT

Analyse des processus menant

à des changements dans une entreprise du secteur aéronautique:

vers un modèle d'évaluation des interventions ergonomiques VALEPAE ALBERT, NICOLE VEZINA, HENRETTE BILODEAN ET FABEEN COUTAREL

Labour Standards in Global Value Chains: Disentangling Workers' Voice, Vicarious Voice, Power Relations, and Regulation

\section{INDUSTRIAL RELATIONS}

\section{QUARTERLY REVIEW}

A bilingual quarterly published since 1945 by the Industrial Relations Department, Université Laval

\section{RI/IR EN LIGNE}

RI/R est disponible en ligne sur le site Erudit :

www.erudit.org/revue/ri

Pour abonnement institutionnel, contacter Érudit.

Pour consulter les règles de publication ou vous abonner, visitez notre site Internet :

www.riir.ulaval.ca

\section{RI/IR ONLINE}

RI/R is available on line on Erudit website at:

www.erudit.org/revue/ri

For an institutional subscription to digitalized issues, please contact Érudit.

Visit our website for Notes to contributors or to subscribe:

www.riir.ulaval.ca

RELATIONS INDUSTRIELLES

INDUSTRIAL RELATIONS

Pavillon J.-A.-DeSéve

1025, avenue des Sciences-Humaines

Bureau 3129, Université Laval

Québec (Québec) Canada G1V 0A6

TELEPHONE : (418) 656-2468

COURRIEL / E-MAIL :

relat.inderlt.ulaval.ca

www.riir.ulaval.ca 